\title{
THE CONVERGENCE ESTIMATES FOR GALERKIN-WAVELET SOLUTION OF PERIODIC PSEUDODIFFERENTIAL INITIAL VALUE PROBLEMS
}

\author{
NGUYEN MINH CHUONG and BUI KIEN CUONG
}

\author{
Received 13 March 2002
}

\begin{abstract}
Using the discrete Fourier transform and Galerkin-Petrov scheme, we get some results on the solutions and the convergence estimates for periodic pseudodifferential initial value problems.
\end{abstract}

2000 Mathematics Subject Classification: 35Sxx, 41A65, 65Txx, 65Mxx.

1. Introduction. In recent years, wavelets have been developing intensively and have become a powerful tool to study mathematics and technology, for example, the theory of the singular integral, singular integro-differential equations, the areas such as sound analysis, image compression, and so on (see $[9,10]$ and references therein). In this paper, we use a scaling function and a multilevel approach to estimate the error of the problem

$$
\begin{gathered}
\frac{\partial u(x, t)}{\partial t}=a \cdot A u(x, t), \quad x \in \mathscr{g}^{n}, t>0, a \in \mathbb{R}, \\
u(x, 0)=\left[u_{0}\right](x), \quad x \in \mathscr{f}^{n},
\end{gathered}
$$

where $A$ is a pseudodifferential operator (see $[1,2,3,4,6,8,9,12]$ ) with a symbol $\sigma \in C^{\infty}\left(\mathbb{R}^{n}\right), \sigma$ is positively homogeneous of degree $r>0$ such that

$$
\left|D^{\alpha} \sigma(\xi)\right| \leq C_{\alpha}(1+|\xi|)^{r-|\alpha|}, \text { for all multi-index } \alpha \in \mathbb{N}^{n}
$$

$g^{n}=\mathbb{R}^{n} / \mathbb{Z}^{n}$, and $\left[u_{0}\right](x)=\sum_{k \in \mathbb{Z}^{n}} u_{0}(x+k)$ is a periodic operator.

We discuss only problem (1.1) with the following condition:

$$
a \sigma(\xi) \leq 0, \quad \forall \xi \in \mathbb{Z}^{n} .
$$

2. Preliminaries and notations. The continuous Fourier transform of the function $f \in L_{2}\left(\mathbb{R}^{n}\right)$ is defined by

$$
\hat{f}(\xi)=\int_{\mathbb{R}^{n}} e^{-2 \pi i x \xi} f(x) d x, \quad \xi \in \mathbb{R}^{n}
$$


with the inverse Fourier formula

$$
f(x)=\int_{\mathbb{R}^{n}} e^{2 \pi i x \xi} \hat{f}(\xi) d \xi, \quad \xi \in \mathbb{R}^{n}
$$

(see $[4,8,11])$.

The discrete Fourier transform of the function $f \in L_{2}\left(g^{n}\right)$ is

$$
\mathscr{F}(f)(\xi)=\tilde{f}(\xi):=\int_{[0,1]^{n}} e^{-2 \pi i x \xi} f(x) d x, \quad \xi \in \mathbb{Z}^{n},
$$

and the inverse Fourier transform is

$$
f(x):=\sum_{\xi \in \mathbb{Z}^{n}} \tilde{f}(\xi) e^{2 \pi i x \xi}
$$

(see [6]).

Some simple properties of the discrete Fourier transform are

$$
(f, g)_{0}=\sum_{\xi \in \mathbb{Z}^{n}} \tilde{f}(\xi) \overline{\tilde{g}(\xi)},
$$

where $(\cdot, \cdot)_{0}$ is the $L_{2}\left(\mathscr{g}^{n}\right)$-inner product,

$$
\|f\|_{0}^{2}=\sum_{\xi \in \mathbb{Z}^{n}}|\tilde{f}(\xi)|^{2}=\|\tilde{f}\|_{l_{2}}^{2},
$$

where $\|\cdot\|_{0}$ is $L_{2}\left(\mathscr{g}^{n}\right)$-norm and $\|\cdot\|_{l_{2}}$ is $l_{2}$-norm.

Let $s \in \mathbb{R}$. Denote

$$
H^{s}\left(\mathscr{F}^{n}\right)=\left\{u \in D^{\prime}\left(\mathscr{F}^{n}\right) \mid\langle D\rangle^{s} u \in L_{2}\left(\mathscr{F}^{n}\right)\right\},
$$

where

$$
\langle\xi\rangle= \begin{cases}1 & \text { if } \xi=0, \\ |\xi| & \text { if } \xi \neq 0\end{cases}
$$

then $H^{s}\left(g^{n}\right)$ is the Sobolev space endowed with the norm

$$
\|u\|_{s}^{2}=\sum_{\xi \in \mathbb{Z}^{n}}\langle\xi\rangle^{2 s}|\tilde{u}(\xi)|^{2}
$$

and the inner product

$$
\langle u, v\rangle_{s}=\sum_{\xi \in \mathbb{Z}^{n}}\langle\xi\rangle^{2 s} \tilde{u}(\xi) \bar{v}(\xi)
$$

Here, we also define the discrete Sobolev space $H_{d}^{s}\left(\mathbb{R}^{n}\right), s \in \mathbb{R}$, of the functions $f \in H^{s}\left(\mathbb{R}^{n}\right)$ such that the following norm is finite:

$$
\|f\|_{s, d}^{2}=\sum_{\xi \in \mathbb{Z}^{n}}\langle\xi\rangle^{2 s}|\hat{f}(\xi)|^{2} .
$$


Denote

$$
\mathscr{L}_{2}=\left\{f \in L_{2}\left(\mathbb{R}^{n}\right): \sum_{\xi \in \mathbb{Z}^{n}}|f(\cdot-\xi)| \in L_{2}\left([0,1]^{n}\right)\right\} .
$$

It is clear that any function $f \in L_{2}\left(\mathbb{R}^{n}\right)$, which has compact support, or any function, for which $\int_{k+[0,1]^{n}}|f(x)|^{2} d x$ decays exponentially as $|k|$ tends to infinity, belongs to $\mathscr{L}_{2}$. The periodic operator [ $u$ ] is totally defined if $u \in \mathscr{L}_{2}$. Here, we assume that $u_{0} \in \mathscr{L}_{2}$.

REMARK 2.1. (1) It follows from (2.1) and (2.3) that if $u \in \mathscr{L}_{2}$, then $\mathscr{F}([u])(\xi)$ $=\hat{u}(\xi), \xi \in \mathbb{Z}^{n}$.

(2) It is clear that if $t \leq s, s, t \in \mathbb{R}$, then $H^{t}\left(\mathscr{g}^{n}\right) \subset H^{s}\left(\mathscr{g}^{n}\right)$.

Using the variable separate method and the discrete Fourier transform, the solution of problem (1.1) can be represented as

$$
u(x, t)=E(t)\left[u_{0}\right](x)=\sum_{\xi \in \mathbb{Z}^{n}} \exp (a \sigma(\xi) t) \mathscr{F}\left(\left[u_{0}\right]\right)(\xi) e^{2 \pi i x \xi},
$$

where $E(t)$ is a differentiable function and $E(0)=1$.

We recall that a multiresolution approximation (MRA) of $L_{2}\left(\mathbb{R}^{n}\right)$ is, as a definition, an increasing sequence $V_{j}, j \in \mathbb{Z}$, of closed linear subspaces of $L_{2}\left(\mathbb{R}^{n}\right)$ with the following properties:

$$
\bigcap_{j \in \mathbb{Z}} V_{j}=\{0\}, \quad \overline{\bigcup_{j \in \mathbb{Z}} V_{j}}=L_{2}\left(\mathbb{R}^{n}\right)
$$

for all $f \in L_{2}\left(\mathbb{R}^{n}\right)$ and all $j \in \mathbb{Z}$,

$$
f(x) \in V_{j} \Longleftrightarrow f(2 x) \in V_{j+1} ;
$$

for all $f \in L_{2}\left(\mathbb{R}^{n}\right)$ and $k \in \mathbb{Z}^{n}$,

$$
f(x) \in V_{0} \Longleftrightarrow f(x-k) \in V_{0} .
$$

There exists a function, called the scaling function (SF) $\phi(x) \in V_{0}$, such that the sequence

$$
\left\{\phi(x-k), k \in \mathbb{Z}^{n}\right\}
$$

is a Riesz basic of $V_{0}$ (see [5, 9]).

An SF $\phi$ is called $\mu$-regular $\left(\mu \in \mathbb{N}\right.$ ) if, for each $m \in \mathbb{N}$, there exists $c_{m}$ such that the following condition holds:

$$
\left|D^{\alpha} \phi(x)\right| \leq c_{m}(1+|x|)^{-m}, \quad \forall \alpha,|\alpha| \leq \mu .
$$


REMARK 2.2. (1) Denote $\phi_{j k}(x)=2^{n j / 2} \phi\left(2^{j} x-k\right), k \in \mathbb{Z}^{n}$. It follows from (2.14), (2.15), (2.16), and (2.17) that $V_{j}=\overline{\operatorname{span}}\left\{\phi_{j k}(x), k \in \mathbb{Z}^{n}\right\}, j \in \mathbb{Z}$.

(2) For each $\mu \in \mathbb{N}$, there exists an SF $\phi(x)$ with compact support, and $\phi(x)$ is $\mu$-regular; so in what follows, we always assume that $\phi$ has compact support and is $\mu$-regular (see [9]).

Using the periodic operator and an MRA of $L_{2}\left(\mathbb{R}^{n}\right)$, we can build an MRA of $L_{2}\left(g^{n}\right)$ with the SF $[\phi]$ as follows.

Denote

$$
\begin{gathered}
\phi_{k}^{j}(x)=2^{n j / 2} \sum_{l \in \mathbb{Z}^{n}} \phi_{j k}(x+l)=2^{n j / 2} \sum_{l \in \mathbb{Z}^{n}} \phi\left(2^{j}(x+l)-k\right), \quad j \geq 0, \\
{\left[V_{j}\right]=\overline{\operatorname{span}}\left\{\phi_{k}^{j}(x), k \in \mathbb{Z}^{n j}\right\}, \quad j \geq 0,}
\end{gathered}
$$

where $\mathbb{Z}^{n j}=\mathbb{Z}^{n} / 2^{j} \mathbb{Z}^{n}$.

Then, the sequence $\left[V_{j}\right]_{j \geq 0}$ satisfies

$$
\left[V_{0}\right] \subset\left[V_{1}\right] \subset \cdots, \quad \overline{\bigcup_{j \geq 0}\left[V_{j}\right]}=L_{2}\left(\mathscr{g}^{n}\right) .
$$

It is clear that $\operatorname{dim}\left[V_{j}\right]=2^{n j}$, and if $\left(\phi_{j k}, \phi_{j l}\right)=\delta_{k l}, k, l \in \mathbb{Z}^{n}$, then $\left(\phi_{k}^{j}, \phi_{l}^{j}\right)=$ $\delta_{k l}, k, l \in \mathbb{Z}^{n j}$ (see [6]).

For each $j \geq 0$, let $P_{j}: L_{2}\left(\mathscr{g}^{n}\right) \rightarrow\left[V_{j}\right]$ be the orthogonal projection from $L_{2}\left(\mathscr{f}^{n}\right)$ on $\left[V_{j}\right]$, which has the following property.

THeOrem 2.3 (see [6, page 600]). Let $-\mu-1 \leq s \leq \mu,-\mu \leq q \leq \mu+1$, and $s \leq q$, then

$$
\left\|u-P_{j} u\right\|_{s} \leq c 2^{j(s-q)}\|u\|_{q}
$$

for all $u \in H^{q}\left(g^{n}\right)$, where $c$ is independent of $j$ and $u$.

Denoting $h=2^{-j}$ and $V_{h}=\left[V_{j}\right]$, we can write (2.22) as

$$
\left\|v-P_{j} v\right\|_{s} \leq c h^{q-s}\|v\|_{q}
$$

3. The Galerkin-wavelet solution. Fix a distribution with compact support $\eta \in H^{-s^{\prime}}(\Gamma)$, where $s^{\prime} \geq 0$ satisfying $A V_{h} \subset H^{s^{\prime}}\left(\mathscr{g}^{n}\right)$ and where $\Gamma \subset \mathbb{R}^{n}$ is some fixed compact domain such as a hypercube. For $f \in H^{s^{\prime}}\left(\mathscr{g}^{n}\right)$, define

$$
\eta_{k}^{j}(f)=2^{-n j / 2} \eta\left(f\left(2^{-j}(\cdot+k)\right)\right) .
$$

The space

$$
X^{j}:=\operatorname{span}\left\{\eta_{k}^{j}, k \in \mathbb{Z}^{n j}\right\}
$$


is contained in $\left(A V_{h}\right)^{\prime}$, which is the dual of $A V_{h}$. The corresponding GalerkinPetrov-wavelet scheme is then given by

$$
\begin{gathered}
\eta_{k}^{j}\left(\frac{\partial u_{h}}{\partial t}\right)=a \eta_{k}^{j}\left(A u_{h}\right), \quad k \in \mathbb{Z}^{n j} \\
u_{h}(x, 0)=R_{h}\left[u_{0}\right](x),
\end{gathered}
$$

where $R_{h} v$ is a linear approximation of $v$ in $V_{h}$ and $u_{h}:[0, \infty) \rightarrow V_{h}$ is a differentiable operator.

Set

$$
\begin{gathered}
u_{h}(x, t)=\sum_{k \in \mathbb{Z}^{n j}} c_{k}(t) \phi_{k}^{j}(x), \\
R_{h}\left[u_{0}\right](x):=\left[u_{0}\right]_{h}(x):=\sum_{k \in \mathbb{Z}^{n j}} c_{k}(0) \phi_{k}^{i}(x) .
\end{gathered}
$$

Then the scheme (3.3) and (3.4) provides an algebra equation system and the solution can be solved by Fourier series.

LEMMA 3.1. The following formulas hold true:

$$
\begin{aligned}
\mathscr{F}\left(\phi_{k}^{j}\right)(\xi) & =h^{n / 2} \hat{\phi}(h \xi) e^{-2 \pi i h k \xi}, \\
\mathscr{F}\left(A \phi_{k}^{j}\right)(\xi) & =h^{n / 2} \sigma(\xi) \hat{\phi}(h \xi) e^{-2 \pi i h k \xi} .
\end{aligned}
$$

Proof. (a) It follows from (2.3) and (2.19) that

$$
\begin{aligned}
\mathscr{F}\left(\phi_{k}^{j}\right)(\xi) & =h^{-n / 2} \sum_{l \in \mathbb{Z}^{n}} \int_{[0,1]^{n}} e^{-2 \pi i x \xi} \phi\left(2^{j}(x+l)-k\right) d x \\
& =h^{n / 2} \sum_{l \in \mathbb{Z}^{n}} \int_{2^{j}\left(l+[0,1]^{n}\right)-k} e^{-2 \pi i h x \xi} \phi(x) d x e^{-2 \pi i k h \xi} \\
& =h^{n / 2} \int_{\mathbb{R}^{n}} e^{-2 \pi i h x \xi} \phi(x) d x e^{-2 \pi i k h \xi} \\
& =h^{n / 2} \hat{\phi}(h \xi) e^{-2 \pi i h k \xi} .
\end{aligned}
$$

(b) We have

$$
\mathscr{F}(A u)(\xi)=\sigma(\xi) \tilde{u}(\xi)
$$

consequently,

$$
\mathscr{F}\left(A \phi_{k}^{j}\right)(\xi)=\sigma(\xi) \mathscr{F}\left(\phi_{k}^{j}\right)(\xi)=h^{n / 2} \sigma(\xi) \hat{\phi}(h \xi) e^{-2 \pi i h k \xi} .
$$

The proof of the lemma is complete. 
COROLLARY 3.2. The following formulas hold true:

$$
\begin{aligned}
\eta_{k}^{j}\left(\phi_{l}^{j}\right) & =h^{n} \sum_{\xi \in \mathbb{Z}^{n}} \hat{\phi}(h \xi) \overline{\hat{\eta}(h \xi)} e^{-2 \pi i h(l-k) \xi} \\
\eta_{k}^{j}\left(A \phi_{l}^{j}\right) & =h^{n} \sum_{\xi \in \mathbb{Z}^{n}} \sigma(\xi) \hat{\phi}(h \xi) \overline{\hat{\eta}(h \xi)} e^{-2 \pi i h(l-k) \xi} .
\end{aligned}
$$

Proof. (a) Using (2.4), Lemma 3.1, and (3.1), we have

$$
\begin{aligned}
\eta_{k}^{j}\left(\phi_{l}^{j}\right) & =\eta_{k}^{j}\left(\sum_{\xi \in \mathbb{Z}^{n}} \mathscr{F}\left(\phi_{l}^{j}\right)(\xi) e^{2 \pi i x \xi}\right) \\
& =\eta_{k}^{j}\left(\sum_{\xi \in \mathbb{Z}^{n}} h^{n / 2} \hat{\phi}(h \xi) e^{-2 \pi i h l \xi} e^{2 \pi i x \xi}\right) \\
& =h^{n} \sum_{\xi \in \mathbb{Z}^{n}} \hat{\phi}(h \xi) e^{-2 \pi h l \xi} \eta\left(e^{2 \pi h(x+k) \xi}\right) \\
& =h^{n} \sum_{\xi \in \mathbb{Z}^{n}} \hat{\phi}(h \xi) \overline{\hat{\eta}(h \xi)} e^{-2 \pi i h(l-k) \xi} .
\end{aligned}
$$

(b) Similarly, we can get the second assertion.

The following lemma is extracted from [6].

LEMMA 3.3. The following formula holds valid:

$$
\sum_{m \in \mathbb{Z}^{n j}} e^{-2 \pi i h m(k-\xi)}= \begin{cases}2^{n j} & \text { if } \xi=k+2^{j} \theta, \theta \in \mathbb{Z}^{n}, \\ 0 & \text { otherwise. }\end{cases}
$$

Set

$$
\begin{aligned}
& \alpha(k)=\sum_{\xi \in \mathbb{Z}^{n}} \hat{\phi}(h \xi) \overline{\hat{\eta}(h \xi)} e^{2 \pi i h k \xi}, \\
& \delta(k)=\sum_{\xi \in \mathbb{Z}^{n}} \sigma(h \xi) \hat{\phi}(h \xi) \overline{\hat{\eta}(h \xi)} e^{2 \pi i h k \xi}, \quad k \in \mathbb{Z}^{n j} .
\end{aligned}
$$

The series

$$
\begin{aligned}
\tilde{\alpha}(\zeta) & =h^{n} \sum_{k \in \mathbb{Z}^{n j}} \alpha(k) e^{-2 \pi i h k \zeta}, \\
\tilde{\delta}(\zeta) & =h^{n} \sum_{k \in \mathbb{Z}^{n j}} \delta(k) e^{-2 \pi i h k \zeta}, \\
\tilde{c}(\zeta, t) & =h^{n} \sum_{k \in \mathbb{Z}^{n j}} c_{k}(t) e^{-2 \pi i h k \zeta}, \quad \zeta \in \mathbb{Z}^{n}
\end{aligned}
$$

are called discrete Fourier series. 
It follows from (3.3), (3.5), the positively homogeneous condition, and Corollary 3.2 that

$$
\sum_{k \in \mathbb{Z}^{n j}} c_{k}^{\prime}(t) \alpha(l-k)=a h^{-r} \sum_{k \in \mathbb{Z}^{n j}} c_{k}(t) \delta(l-k), \quad l \in \mathbb{Z}^{n j} .
$$

Thus

$$
\begin{aligned}
\tilde{c}_{t}^{\prime}(\zeta, t) \tilde{\alpha}(\zeta) & =a h^{-r} \tilde{c}(\zeta, t) \tilde{\delta}(\zeta) \\
\tilde{c}(\zeta, t) & =\exp \left(\frac{a t}{h^{r}} \frac{\tilde{\delta}(\zeta)}{\tilde{\alpha}(\zeta)}\right) \tilde{c}(\zeta, 0) .
\end{aligned}
$$

For each $\tau=0,1$, set

$$
g_{\phi, \tau}(\zeta)=\sum_{k \in \mathbb{Z}^{n}} \sigma(h \zeta+k)^{\tau} \hat{\phi}(h \zeta+k) \overline{\hat{\eta}(h \zeta+k)} .
$$

LEMMA 3.4. If the series (3.22) converges absolutely, then

$$
\tilde{\alpha}(\zeta)=g_{\phi, 0}(\zeta), \quad \tilde{\delta}(\zeta)=g_{\phi, 1}(\zeta)
$$

Proof. (a) From (3.14) and (3.16), it follows that

$$
\tilde{\alpha}(\zeta)=h^{n} \sum_{k \in \mathbb{Z}^{n j}} \sum_{\xi \in \mathbb{Z}^{n}} \hat{\phi}(h \xi) \overline{\hat{\eta}(h \xi)} e^{-2 \pi i h k(\zeta-\xi)} .
$$

By the hypothesis of the lemma, we can interchange the summation in the above double sum; then by using the variable change and Lemma 3.3, it is easy to see that

$$
\begin{aligned}
\tilde{\alpha}(\zeta) & =h^{n} \sum_{\xi \in \mathbb{Z}^{n}} \hat{\phi}(h \xi) \overline{\hat{\eta}(h \xi)} \sum_{k \in \mathbb{Z}^{n j}} e^{-2 \pi i h k(\zeta-\xi)} \\
& =\sum_{\theta \in \mathbb{Z}^{n}} \hat{\phi}(h \zeta+\theta) \overline{\hat{\eta}(h \zeta+\theta)}=g_{\phi, 0}(\zeta) .
\end{aligned}
$$

(b) Similarly, the second assertion of the lemma will be checked.

From (3.5), (3.6), and (3.21), it follows that

$$
\tilde{u}_{h}(\xi, t)=\exp \left(\frac{a t}{h^{r}} \frac{\tilde{\delta}(\xi)}{\tilde{\alpha}(\xi)}\right) \mathscr{F}\left(\left[u_{0}\right]_{h}\right)(\xi) .
$$

Let $F_{h}(t)$ be the operator defined by

$$
\mathscr{F}\left(F_{h}(t) v(\cdot)\right)(\xi)=\exp \left(\frac{a t}{h^{r}} \frac{\tilde{\delta}(\xi)}{\tilde{\alpha}(\xi)}\right) \tilde{v}(\xi),
$$

then the approximation $u_{h}(x)$ can be represented by

$$
u_{h}(x)=F_{h}(t) R_{h}\left[u_{0}\right](x) .
$$


4. The error estimate of approximation solutions. Now to estimate the error, we need some restrictions on the $\sigma, \phi$, and $\eta$ used above. The triplet $(\sigma, \phi, \eta)$ is called admissible if the following properties hold:

(i) there exists $p \in \mathbb{N}, p \geq r$, such that the series

$$
\sum_{k \in \mathbb{Z}^{n}} \sigma(h \xi+k) \hat{\phi}(h \xi+k) \overline{\hat{\eta}(h \xi+k)}
$$

converges absolutely and

$$
\sum_{k \in \mathbb{Z}^{n}} \sigma(h \xi+k) \hat{\phi}(h \xi+k) \overline{\hat{\eta}(h \xi+k)}=\sigma(h \xi) \hat{\phi}(h \xi) \overline{\hat{\eta}(h \xi)}+o\left(|h \xi|^{p}\right)
$$

as $|h \xi| \rightarrow 0$,

(ii) $\hat{\phi}(\xi) \overline{\hat{\eta}(\xi)} \geq 0$, for all $\xi \in \mathbb{R}^{n}, \hat{\phi}(0) \overline{\hat{\eta}(0)} \neq 0$,

(iii) the series

$$
\sum_{k \in \mathbb{Z}^{n}} \hat{\phi}(h \xi+k) \overline{\hat{\eta}(h \xi+k)}
$$

converges and

$$
\sum_{k \in \mathbb{Z}^{n}} \hat{\phi}(h \xi+k) \overline{\hat{\eta}(h \xi+k)}=\hat{\phi}(h \xi) \overline{\hat{\eta}(h \xi)}+0\left(|h \xi|^{p}\right)
$$

as $|h \xi| \rightarrow 0$.

REMARK 4.1. (1) If $\eta=\phi$ and $\sigma$ is a pseudodifferential operator with symbol $\sigma(\xi)=|\xi|^{r}, 0<r \leq \mu$, then the triplet $(\sigma, \phi, \phi)$ is automatically admissible at least for $p=\mu$, where $\mu \in \mathbb{N}$ is used in (2.18) (see [7] for detail).

(2) If $\eta=\phi$ and $\sigma$ is a pseudodifferential operator with symbol $\sigma(\xi)=\langle\xi\rangle^{2}$, then the triplet $\left(\langle\xi\rangle^{2}, \phi, \phi\right)$ is admissible for $p=\mu$ (see [6]).

Write

$$
u-u_{h}=\left\{u-F_{h}(t)\left[u_{0}\right]\right\}+F_{h}(t)\left\{\left[u_{0}\right]-R_{h}\left[u_{0}\right]\right\}
$$

We have

$$
\begin{aligned}
\mathscr{F}\left(F_{h}(t)\left[u_{0}\right](\cdot)\right)(\xi) & =\exp \left(\frac{a t}{h^{r}} \frac{\tilde{\delta}(\xi)}{\tilde{\alpha}(\xi)}\right) \mathscr{F}\left(\left[u_{0}\right]\right)(\xi) \\
& =\exp \left(\frac{a t}{h^{r}} \frac{\tilde{\delta}(\xi)}{\tilde{\alpha}(\xi)}\right) \hat{u}_{0}(\xi), \quad \xi \in \mathbb{Z}^{n}
\end{aligned}
$$

thus

$$
\begin{aligned}
\mathscr{F}(u & \left.-F_{h}(t)\left[u_{0}\right]\right)(\xi) \\
& =\left\{\exp (a t \sigma(\xi))-\exp \left(\frac{a t}{h^{r}} \frac{\tilde{\delta}(\xi)}{\tilde{\alpha}(\xi)}\right)\right\} \hat{u}_{0}(\xi), \quad \xi \in \mathbb{Z}^{n} .
\end{aligned}
$$


If the triplet $(\sigma, \phi, \eta)$ is admissible, then it follows from (3.22) and Lemma 3.4 that

$$
\frac{\tilde{\delta}(\xi)}{\tilde{\alpha}(\xi)}=\sigma(h \xi)+0\left(|h \xi|^{p}\right) \quad \text { as }|h \xi| \longrightarrow 0
$$

THEOREM 4.2. Suppose that $r+s^{\prime} \leq s \leq p, 0 \leq m \leq s$, and it is assumed that the triplet $(\sigma, \phi, \eta)$ is admissible. Then, for $u_{0} \in \mathscr{L}_{2} \cap H_{d}^{m+s}\left(\mathbb{R}^{n}\right), 0 \leq t \leq T$, with $h$ small enough, we get

$$
\left\|u-F_{h}(t)\left[u_{0}\right]\right\|_{m} \leq c h^{s-r}\left\|u_{0}\right\|_{s+m, d}
$$

where $c$ is independent of $u, h$, and $u_{0}$.

PROOF. It follows from (4.8) that

$$
\left|\operatorname{at} \sigma(\xi)-\frac{a t}{h^{r}} \frac{\tilde{\delta}(\xi)}{\tilde{\alpha}(\xi)}\right| \leq c h^{p-r}|\xi|^{p} \quad \text { as }|h \xi| \leq 1 .
$$

The equality

$$
e^{t a}-e^{t b}=t(a-b) \int_{0}^{1} e^{s t a+(1-s) t b} d s
$$

(4.10), and (1.3) imply that, for $r \leq s \leq p$ and $0 \leq t \leq T$,

$$
\left|\exp (\operatorname{at} \sigma(\xi))-\exp \left(\frac{a t}{h^{r}} \frac{\tilde{\delta}(\xi)}{\tilde{\alpha}(\xi)}\right)\right| \leq c h^{s-r}|\xi|^{s} \quad \text { as }|h \xi| \leq 1
$$

Hence, from (4.7) and (4.12), we obtain

$$
\left|\mathscr{F}\left(u(\cdot, t)-F_{h}(t)\left[u_{0}\right](\cdot)\right)(\xi)\right| \leq c h^{s-r}|\xi|^{s}\left|\hat{u}_{0}(\xi)\right| \quad \text { as }|h \xi| \leq 1 .
$$

By (1.3) and the admissibility of the triplet $(\sigma, \phi, \eta)$, inequality (4.13) is also valid for all $\xi \in \mathbb{Z}^{n}$. Hence, for each $0 \leq m \leq s, r+s^{\prime} \leq s \leq p$, and $0 \leq t \leq T$, we get

$$
\begin{aligned}
\left\|u-F_{h}(t)\left[u_{0}\right]\right\|_{m}^{2} & =\sum_{\xi \in \mathbb{Z}^{n}}\langle\xi\rangle^{2 m}\left|\mathscr{F}\left\{u(\cdot, t)-F_{h}(t)\left[u_{0}\right](\cdot)\right\}(\xi)\right|^{2} \\
& \leq c h^{2(s-r)} \sum_{\xi \in \mathbb{Z}^{n}}\langle\xi\rangle^{2(m+s)}\left|\hat{u}_{0}(\xi)\right|^{2} \\
& \leq c h^{2(s-r)}\left\|u_{0}\right\|_{m+s, d}^{2} .
\end{aligned}
$$

The theorem is thus proved. 
From the admissibility of the triplet $(\sigma, \phi, \eta)$ and (1.3), it follows that $F_{h}(t)$ : $H^{m}\left(\mathbb{R}^{n}\right) \rightarrow H^{m}\left(\mathbb{R}^{n}\right), 0 \leq m \leq s$, is a continuous linear operator. Consequently,

$$
\left\|F_{h}(t)\left(\left[u_{0}\right]-R_{h}\left[u_{0}\right]\right)\right\|_{m} \leq c\left\|\left[u_{0}\right]-R_{h}\left[u_{0}\right]\right\|_{m} .
$$

Therefore, if we assume that

$$
\left\|\left(I-R_{h}\right)\left[u_{0}\right]\right\|_{m} \leq c h^{s}\left\|\left[u_{0}\right]\right\|_{m+s},
$$

then

$$
\left\|F_{h}(t)\left(\left[u_{0}\right]-R_{h}\left[u_{0}\right]\right)\right\|_{m} \leq c h^{s}\left\|\left[u_{0}\right]\right\|_{m+s}
$$

REMARK 4.3. It follows from (2.23) that the assumption (4.17) is satisfied, when $R_{h}=P_{j}$ for $0 \leq m, m+s \leq \mu+1$.

Thus from (4.5), (4.9), and (4.17), we obtain the following theorem.

THEOREM 4.4. If all the hypotheses of Theorem 4.2 and assumption (4.17) are satisfied, then

$$
\left\|u-u_{h}\right\|_{m} \leq c h^{s-r}\left\|u_{0}\right\|_{m+s, d}+c h^{s}\left\|\left[u_{0}\right]\right\|_{m+s}
$$

where $c$ is independent of $u_{0}, h$.

ACKNOWLEDGMENT. The authors thank the referee and the managing editor for their helpful comments and suggestions.

\section{REFERENCES}

[1] N. M. Chuong, Parabolic pseudodifferential operators of variable order, Dokl. Akad. Nauk SSSR 258 (1981), no. 6, 1308-1312.

[2] __ Parabolic systems of pseudo differential equations of variable order, Dokl. Akad. Nauk SSSR 264 (1982), no. 2, 299-302.

[3] _ Degenerate parabolic pseudo differential operator of variable order, Dokl. Akad. Nauk SSSR 268 (1983), no. 5, 1055-1058.

[4] N. M. Chuong, N. M. Tri, and L. Q. Trung, Theory of Partial Differential Equations, Science and Technology Publishing House, Hanoi, 1995 (Vietnamese).

[5] N. M. Chuong and T. N. Tri, The integral wavelet transform in $L^{p}\left(\mathbf{R}^{n}\right), 1 \leq p \leq \infty$, Fract. Calc. Appl. Anal. 3 (2000), no. 2, 133-140.

[6] W. Dahmen, S. Prössdorf, and R. Schneider, Wavelet approximation methods for pseudodifferential equations. I. Stability and convergence, Math. Z. 215 (1994), no. 4, 583-620.

[7] S. M. Gomes and E. Cortina, Convergence estimates for the wavelet Galerkin method, SIAM J. Numer. Anal. 33 (1996), no. 1, 149-161.

[8] L. Hörmander, The Analysis of Linear Partial Differential Operators. I. Distribution Theory and Fourier Analysis, Grundlehren der Mathematischen Wissenschaften, vol. 256, Springer-Verlag, Berlin, 1983.

[9] Y. Meyer, Ondelettes et opérateurs. I [Wavelets and Operators. I], Actualités Mathématiques, Hermann, Paris, 1990 (French). 
[10] , Oscillating Patterns in Image Processing and Nonlinear Evolution Equations. The Fifteenth Dean Jacqueline B. Lewis Memorial Lectures, University Lecture Series, vol. 22, American Mathematical Society, Rhode Island, 2001.

[11] E. M. Stein and G. Weiss, Introduction to Fourier Analysis on Euclidean Spaces, Princeton University Press, New Jersey, 1975.

[12] F. Trèves, Introduction to Pseudodifferential and Fourier Integral Operators, I, II, Plenum Press, New York, 1982.

Nguyen Minh Chuong: National Centre for Natural Science and Technology, Institute of Mathematics, 18 Hoang Quoc Viet Road, Cau Giay District, Hanoi, Vietnam

E-mail address: nmchuong@thevinh.ncst.ac.vn

Bui Kien Cuong: Department of Mathematics, Hanoi Pedagogical University, Number 2, Xuan Hoa, Me Linh, Vinh Phu, Vietnam

E-mail address: bkhcuong@hn.vnn.vn 


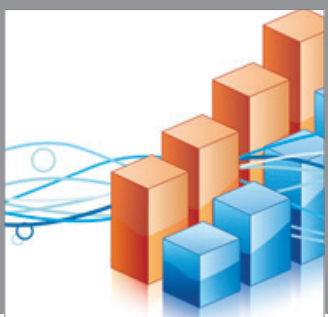

Advances in

Operations Research

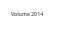

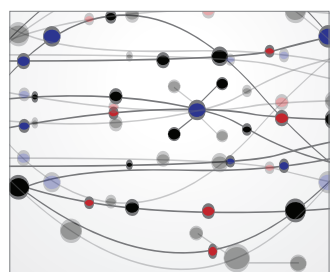

\section{The Scientific} World Journal
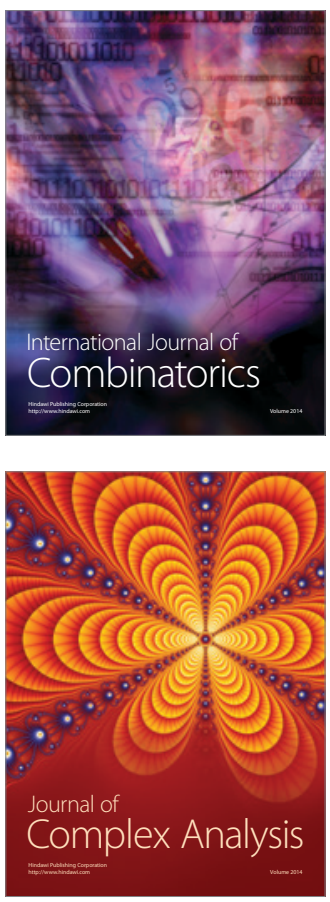

International Journal of

Mathematics and

Mathematical

Sciences
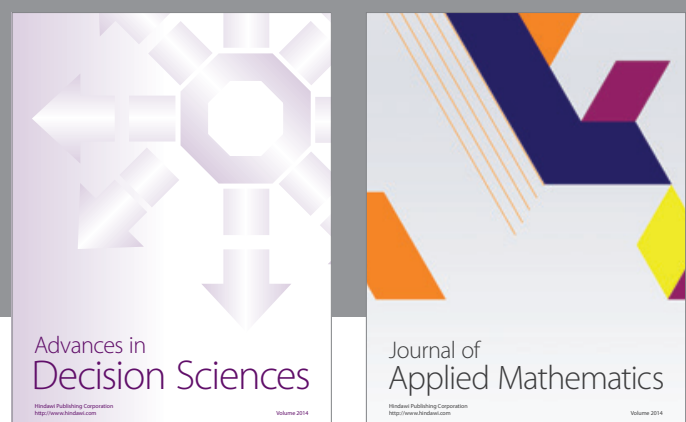

Journal of

Applied Mathematics
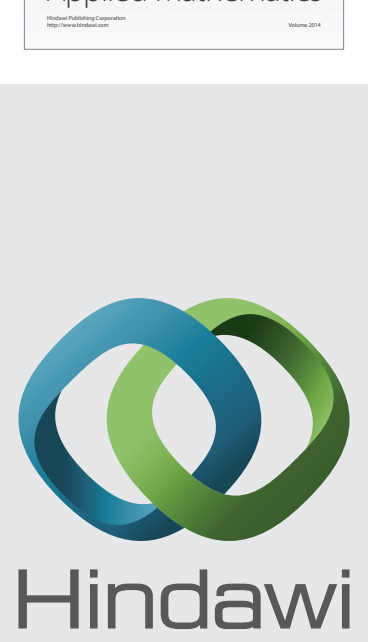

Submit your manuscripts at http://www.hindawi.com
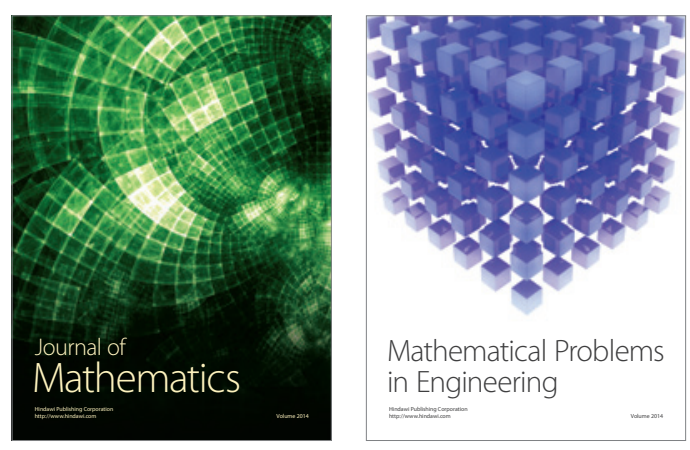

Mathematical Problems in Engineering
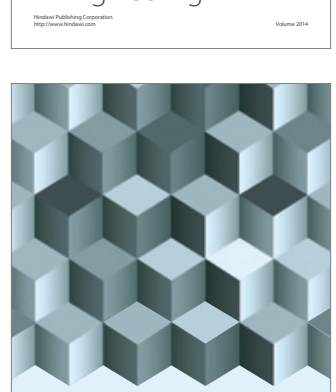

Journal of

Function Spaces
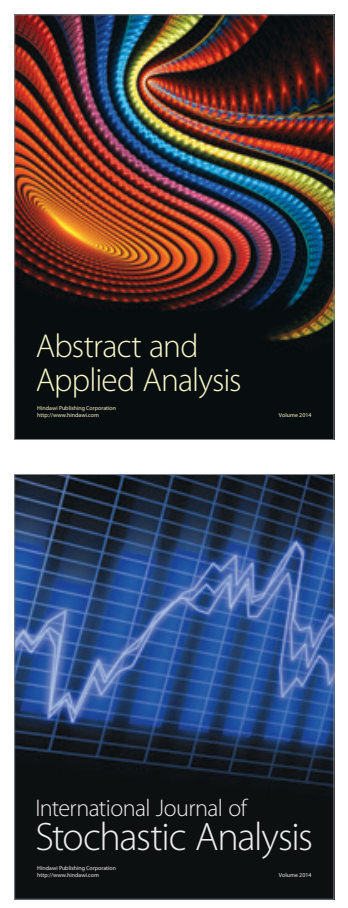

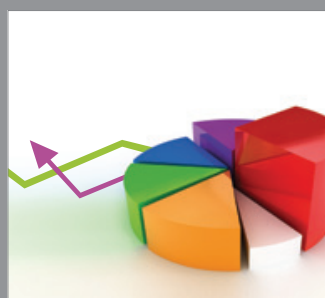

ournal of

Probability and Statistics

Promensencen
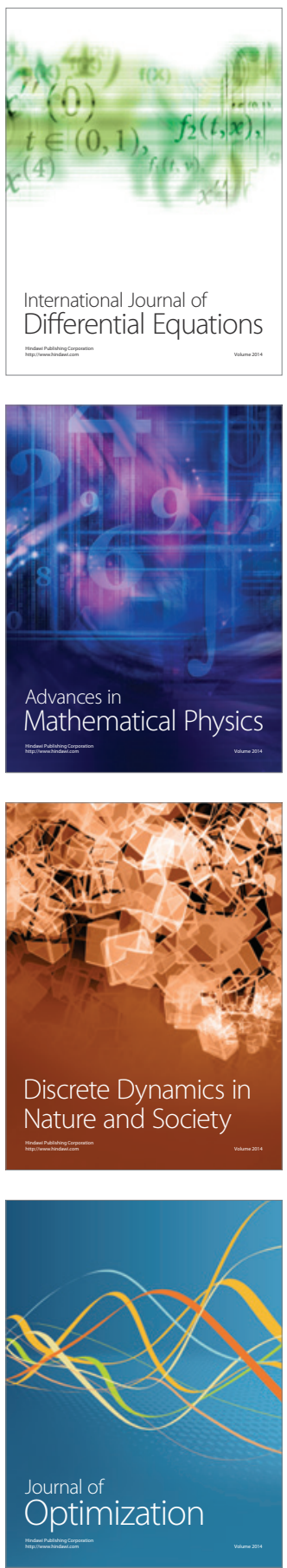